\title{
Some Uniqueness Results of Q-Shift Difference Polynomials Involving Sharing Functions*
}

\author{
Xuexue Qian, Yasheng Ye \\ Department of Mathematics, College of Sciences, University of Shanghai for Science and Technology, Shanghai, China \\ Email: 1714774700@qq.com, yashengye@aliyun.com
}

How to cite this paper: Qian, X.X. and Ye, Y.S. (2017) Some Uniqueness Results of Q-Shift Difference Polynomials Involving Sharing Functions. Applied Mathematics, 8, 1117-1127.

https://doi.org/10.4236/am.2017.88084

Received: July 26, 2017

Accepted: August 18, 2017

Published: August 21, 2017

Copyright $\odot 2017$ by authors and Scientific Research Publishing Inc. This work is licensed under the Creative Commons Attribution International License (CC BY 4.0).

http://creativecommons.org/licenses/by/4.0/

\section{(c) (i) Open Access}

\begin{abstract}
In this paper, we mainly study the uniqueness of specific q-shift difference polynomials $f^{n}(z) \prod_{j=1}^{d} f\left(q_{j} z+c_{j}\right)^{v_{j}} \quad$ and $g^{n}(z) \prod_{j=1}^{d} g\left(q_{j} z+c_{j}\right)^{v_{j}} \quad$ of meromorphic functions, which share a common small function and get the corresponding results. In addition, we also investigate the problem of value distribution on q-shift difference polynomials of entire functions.
\end{abstract}

\section{Keywords}

Value Distribution, Meromorphic Functions, Difference Polynomials, Uniqueness

\section{Introduction}

In recent years, many Scholars have been interested in value distribution of difference operators of meromorphic functions (see [1]-[6]). Furthermore, a large number of papers have studied and obtained the uniqueness results of difference polynomials of meromorphic functions, their shifts and difference operators (see [7]-[12]). Our purpose in the paper is to study the value distribution for q-shift polynomials of transcendental meromorphic with zero order, and some results about entire functions.

For a meromorphic function $f$, we always assume that $f$ is meromorphic in the complex plane $\mathbb{C}$. We use standard notations of the Nevanlinna Value Distribution Theory (see [13]), such as $m(r, f), N(r, f), \bar{N}(r, f), T(r, f)$, $S(r, f)$, and define $N_{2}\left(r, \frac{1}{f}\right)$ as the counting function of zero of $f$, such that simple zero is counted once and multiple zeros are counted twice. We denote any quantity by $S(r, f)$, if it satisfies $S(r, f)=o(T(r, f))$, as $r \rightarrow \infty$

*Supported by the National Natural Science Foundation of China (No.11371139). 
outside of a possible exceptional set of $r$ with finite logarithmic measure. In addition, the notation $\rho(f)$ is the order of growth of $f$. Let meromorphic function $\alpha$ be a common small function of $f(z)$ and $g(z)$, suppose that $f(z)-\alpha(z)$ and $g(z)-\alpha(z)$ have the same zeros counting multiplicities (ignoring multiplicities), then we say that $f$ and $g$ share $\alpha(z) \operatorname{CM}(\mathrm{IM})$.

In this paper, we define a q-shift difference product of meromorphic function $f(z)$ as follows.

$$
\begin{gathered}
F(z)=f^{n}(z) \prod_{j=1}^{d} f\left(q_{j} z+c_{j}\right)^{v_{j}} \\
F_{1}(z)=P_{n}(f(z)) \prod_{j=1}^{d} f\left(q_{j} z+c_{j}\right)^{v_{j}}
\end{gathered}
$$

where $c_{j} \in \mathbb{C} \quad\left(c_{j} \neq 0, j=1,2,3, \cdots, d\right)$ are distinct constants, $q_{j}(j=1,2, \cdots, d)$ be non-zero finite complex constants, let $P_{n}(z)=\alpha_{n} z^{n}+\alpha_{n-1} z^{n-1}+\cdots+\alpha_{1} z+\alpha_{0}$ be a non-zero polynomial, where $\alpha_{n}(\neq 0), \alpha_{n-1}, \cdots, \alpha_{0}$ are small functions of $f$. Let $n, d, v_{j}(j=1,2, \cdots, d)$ are positive integers and $\sigma=v_{1}+v_{2}+\cdots+v_{d}$.

Recently, Liu et al. [14] have considered and proved the uniqueness of q-shift difference polynomials of meromorphic functions.

Theorem A. Let $f(z)$ and $g(z)$ be two transcendental meromorphic functions with $\rho(f)=\rho(g)=0$. Let $q$ and $\eta$ be two non-zero finite complex constants. If $f^{n}(z) f(q z+\eta)$ and $g^{n}(z) g(q z+\eta)$ share $1 \mathrm{CM}$, then either $f(z)=\operatorname{tg}(z)$ or $f(z) g(z)=t$, where $n\left(\in N^{*}\right) \geq 14$ satisfying $t^{n+1}=1$.

Theorem B. Let $f(z)$ and $g(z)$ be two transcendental meromorphic functions with $\rho(f)=\rho(g)=0$. Let $q$ and $\eta$ be two non-zero finite complex constants. If $f^{n}(z) f(q z+\eta)$ and $g^{n}(z) g(q z+\eta)$ share 1 IM, then either $f(z)=\operatorname{tg}(z)$ or $f(z) g(z)=t$, where $n\left(\in N^{*}\right) \geq 26 \quad$ satisfying $t^{n+1}=1$

First, we will prove the following theorems on value sharing results of q-shift difference polynomials extend the Theorem A, B, as follows:

Theorem 1.1. Let $f(z)$ and $g(z)$ be two transcendental meromorphic functions with $\rho(f)=\rho(g)=0$, and let $\alpha(z)(\not \equiv 0)$ be a common small function of $f(z)$ and $g(z)$. If $F(z)$ and $G(z)$ share $\alpha(z) C M$, then $f(z)=\operatorname{tg}(z)$, where $n \geq 4 \min (2 d, \sigma)+\sigma+9$ satisfying $t^{n+\sigma}=1$.

Theorem 1.2. Let $f(z)$ and $g(z)$ be two transcendental meromorphic functions with $\rho(f)=\rho(g)=0$, and let $\alpha(z)(\equiv 0)$ be a common small function of $f(z)$ and $g(z)$. If $F(z)$ and $G(z)$ share $\alpha(z)$ IM, then $f(z)=\operatorname{tg}(z)$, where $n \geq 4 \min (2 d, \sigma)+\sigma+6 d+15$ satisfying $t^{n+\sigma}=1$.

Liu et al. [14] also considered some properties of q-shift difference polynomials of entire functions, as follow:

Theorem C. Let $f(z)$ and $g(z)$ be two transcendental entire functions with $\rho(f)=\rho(g)=0$, and let $q$ and $\eta$ are two non-zero finite complex constants, and let $P_{n}(z)=\alpha_{n} z^{n}+\alpha_{n-1} z^{n-1}+\cdots+\alpha_{1} z+\alpha_{0}$ be a non-zero polynomial, where $\alpha_{n}(\neq 0), \alpha_{n-1}, \cdots, \alpha_{0}$, are constants, and let $\mathrm{m}$ be the number 
of the distinct zero of $P_{n}(z)$. If $P_{n}(f(z)) f(q z+\eta)$ and $P_{n}(g(z)) g(q z+\eta)$ share $1 \mathrm{CM}$, then only one of the following two cases holds:

a) $f(z)=\operatorname{tg}(z)$, where $n>2 m+1$, and $k$ is greatest common divisor of $\left(\lambda_{0}, \lambda_{1}, \cdots, \lambda_{n}\right)$, satisfying $t^{k}=1$. When $\alpha_{i}=0$, then $\lambda_{i}=n+1$, otherwise $\lambda_{i}=i+1 . \quad i=0,1, \cdots, n$.

b) $f(z)$ and $g(z)$ satisfy a algebraic equation $Q(f(z), g(z))=0$, where

$$
Q\left(w_{1}, w_{2}\right)=P_{n}\left(w_{1}\right) w_{1}(q z+c)-P_{n}\left(w_{2}\right) w_{2}(q z+c)
$$

Next, it is easy to derive that $P_{n}(f(z)) f(q z+\eta)$ in Theorem $C$ can be replaced by $P_{n}(f(z)) \prod_{j=1}^{d} f\left(q_{j} z+c_{j}\right)^{v_{j}}$, as follows

Theorem 1.3. Let $f(z)$ and $g(z)$ be two transcendental entire functions with $\rho(f)=\rho(g)=0$, and let $\alpha(z)$ be a common small function of $f(z)$ and $g(z)$, and let $k$ be the number of distinct zeros of $P_{n}(z)$. If $F_{1}(z)$ and $G_{1}(z)$ share $\alpha(z) \mathrm{CM}$, then only one of the following results holds:

a) $f(z)=\operatorname{tg}(z)$ for a constant $t$ such that $t^{m}=1$, where $n>2 k+2 d+\sigma$ and $m$ is greatest common divisor of $(n+\sigma, n+\sigma-1, \cdots, n+\sigma-i, \cdots, \sigma+1)$, $\alpha_{n-i} \neq 0, \quad i=0,1, \cdots, n-1$.

b) $f(z)$ and $g(z)$ satisfy a algebraic equation $Q(f, g) \equiv 0$, where

$$
Q\left(w_{1}, w_{2}\right)=P_{n}\left(w_{1}\right) \prod_{j=1}^{d} w_{1}\left(q_{j} z+c_{j}\right)^{v_{j}}-P_{n}\left(w_{2}\right) \prod_{j=1}^{d} w_{2}\left(q_{j} z+c_{j}\right)^{v_{j}} .
$$

\section{Some Lemmas}

Lemma 2.1. (see [15]) Let $n(\geq 1)$ be a positive integer, and let $f(z)$ be a transcendental meromorphic function, and let $\alpha_{i}(i=0,1, \cdots, n)$ be small meromorphic functions of $f$. If

$$
P_{n}(f(z))=\alpha_{n} f^{n}(z)+\alpha_{n-1} f^{n-1}(z)+\cdots+\alpha_{1} f(z)+\alpha_{0},
$$

then

$$
T\left(r, P_{n}(f(z))\right)=n T(r, f(z))+S(r, f(z))
$$

Lemma 2.2. (see [9]) Let $q$ and $\eta$ be two non-zero finite complex numbers, and let $f(z)$ be a nonconstant meromorphic function with $\rho(f)=0$, then

$$
m\left(r, \frac{f(q z+\eta)}{f(z)}\right)=S(r, f) .
$$

on a set of logarithmic density 1 .

Lemma 2.3. (see [12]) Let $f(z)$ and $g(z)$ be two non-constant meromorphic functions. Let $f(z)$ and $g(z)$ share 1 IM and

$$
L=\frac{f^{\prime \prime}}{f^{\prime}}-2 \frac{f^{\prime}}{f-1}-\frac{g^{\prime \prime}}{g^{\prime}}+2 \frac{g^{\prime}}{g-1}
$$

If $L \not \equiv 0$, then 


$$
\begin{aligned}
& T(r, f)+T(r, g) \\
& \leq 2\left(N_{2}(r, f)+N_{2}(r, g)+N_{2}\left(r, \frac{1}{f}\right)+N_{2}\left(r, \frac{1}{g}\right)\right) \\
& \quad+3\left(\bar{N}(r, f)+\bar{N}(r, g)+\bar{N}\left(r, \frac{1}{f}\right)+\bar{N}\left(r, \frac{1}{g}\right)\right)+S(r, f)+S(r, g)
\end{aligned}
$$

Lemma 2.4. (see [16]) Let $f$ and $g$ be two non-constant meromorphic functions. If $f$ and $g$ share $1 \mathrm{CM}$, then only one of the following results holds:

(a) $\max \{T(r, f), T(r, g)\}$

$$
\leq N_{2}(r, f)+N_{2}(r, g)+N_{2}\left(r, \frac{1}{f}\right)+N_{2}\left(r, \frac{1}{g}\right)+S(r, f)+S(r, g)
$$

(b) $f \equiv g$;

(c) $f g \equiv 1$.

Lemma 2.5. (see [14]) Let $q$ and $\eta$ be two non-zero finite complex constants, and let $f$ be a non-constant meromorphic function with $\rho(f)=0$, then

$$
T(r, f(q z+\eta)) \leq T(r, f(z))+S(r, f)
$$

on a set of logarithmic density 1 .

Lemma 2.6. (see [14]) Let $q$ and $\eta$ be two non-zero finite complex constants, and let $f$ be a nonconstant meromorphic function of zero order, then

$$
\begin{aligned}
& \bar{N}(r, f(q z+\eta)) \leq \bar{N}(r, f(z))+S(r, f) \\
& \bar{N}\left(r, \frac{1}{f(q z+\eta)}\right) \leq \bar{N}\left(r, \frac{1}{f(z)}\right)+S(r, f) \\
& N(r, f(q z+\eta)) \leq N(r, f(z))+S(r, f) \\
& N\left(r, \frac{1}{f(q z+\eta)}\right) \leq N\left(r, \frac{1}{f(z)}\right)+S(r, f) .
\end{aligned}
$$

Lemma 2.7. Let $f(z)$ be a non-constant meromorphic function of zero order, and $F_{1}(z)$ be defined as in (2). Then

$$
(n-\sigma) T(r, f)+S(r, f) \leq T\left(r, F_{1}\right) \leq(n+\sigma) T(r, f)+S(r, f)
$$

Proof. Combining Lemma 2.1 with Lemma 2.5, we obtain

$$
\begin{aligned}
T\left(r, F_{1}\right) & \leq T\left(r, P_{n}(f(z))\right)+T\left(r, \prod_{j=1}^{d} f\left(q_{j} z+c_{j}\right)^{v_{j}}\right)+S(r, f) \\
& \leq n T(r, f(z))+\sum_{j=1}^{d} T\left(r, f\left(q_{j} z+c_{j}\right)^{v_{j}}\right)+S(r, f) \\
& \leq(n+\sigma) T(r, f(z))+S(r, f)
\end{aligned}
$$

In addition, by Lemma 2.1 and Lemma 2.5 , we also get 


$$
\begin{aligned}
& (n+\sigma) T(r, f(z)) \leq T\left(r, P_{n}(f(z)) f^{\sigma}\right)+S(r, f) \\
& =m\left(r, P_{n}(f(z)) f^{\sigma}\right)+N\left(r, P_{n}(f(z)) f^{\sigma}\right)+S(r, f) \\
& \leq m\left(r, \frac{F_{1}(z) f^{\sigma}}{\prod_{j=1}^{d} f\left(q_{j} z+c_{j}\right)^{v_{j}}}\right)+N\left(r, \frac{F_{1}(z) f^{\sigma}}{\prod_{j=1}^{d} f\left(q_{j} z+c_{j}\right)^{v_{j}}}\right)+S(r, f) \\
& \leq m\left(r, F_{1}\right)+N\left(r, F_{1}\right)+T\left(r, \frac{f^{\sigma}}{\prod_{j=1}^{d} f\left(q_{j} z+c_{j}\right)^{v_{j}}}\right)+S(r, f) \\
& \leq T\left(r, F_{1}\right)+2 \sigma T(r, f)+S(r, f)
\end{aligned}
$$

which is equivalent to

$$
(n-\sigma) T(r, f)+S(r, f) \leq T\left(r, F_{1}\right)
$$

Therefore, we get Lemma 2.7 .

Lemma 2.8. Let $f(z)$ be an entire function with $\rho(f)=0$, and $F_{1}(z)$ be stated as in (2). Then

$$
T\left(r, F_{1}\right)=(n+\sigma) T(r, f)+S(r, f)
$$

Proof. Using the same method as the Lemma 2.7, we can easily to prove.

\section{Proof of Theorem}

\subsection{Proof of Theorem 1.1}

Set $F^{*}(z)=\frac{F(z)}{\alpha(z)}, G^{*}(z)=\frac{G(z)}{\alpha(z)}$, than $F^{*}(z)$ and $G^{*}(z)$ share $1 \mathrm{CM}$.

Thus by Nevanlinna second fundamental theory, Lemma 2.5 and Lemma 2.7, we have

$$
\begin{aligned}
& (n-\sigma) T(r, f)+S(r, f) \leq T\left(r, F^{*}(z)\right) \\
& \leq \bar{N}\left(r, F^{*}(z)\right)+\bar{N}\left(r, \frac{1}{F^{*}(z)}\right)+\bar{N}\left(r, \frac{1}{F^{*}(z)-1}\right)+S\left(r, F^{*}(z)\right) \\
& \leq \bar{N}\left(r, f^{n}\right)+\bar{N}\left(r, \prod_{j=1}^{d} f\left(q_{j} z+c_{j}\right)^{v_{j}}\right)+\bar{N}\left(r, \frac{1}{f^{n}}\right) \\
& +\bar{N}\left(r, \frac{1}{\prod_{j=1}^{d} f\left(q_{j} z+c_{j}\right)^{v_{j}}}\right)+\bar{N}\left(r, \frac{1}{G^{*}(z)-1}\right)+S(r, f) \\
& \leq(2 d+2) T(r, f)+(n+\sigma) T(r, g)+S(r, g)+S(r, f)
\end{aligned}
$$

Then

$$
(n-2 d-\sigma-2) T(r, f) \leq(n+\sigma) T(r, g)+S(r, g)+S(r, f)
$$

Similarly,

$$
(n-2 d-\sigma-2) T(r, g) \leq(n+\sigma) T(r, f)+S(r, f)+S(r, g)
$$


It follows that $S(r, f)=S(r, g)$.

Then by Lemma 2.4, we consider three subcases.

Case 1. Suppose that

$$
\begin{aligned}
& \max \left\{T\left(r, F^{*}(z)\right), T\left(r, G^{*}(z)\right)\right\} \leq N_{2}\left(r, F^{*}(z)\right)+N_{2}\left(r, \frac{1}{F^{*}(z)}\right)+N_{2}\left(r, G^{*}(z)\right) \\
& +N_{2}\left(r, \frac{1}{G^{*}(z)}\right)+S\left(r, F^{*}(z)\right)+S\left(r, G^{*}(z)\right)
\end{aligned}
$$

holds.

Through simple calculation, we have

$$
\begin{aligned}
& N_{2}\left(r, F^{*}(z)\right) \leq N_{2}\left(r, f^{n}\right)+N_{2}\left(r, \prod_{j=1}^{d} f\left(q_{j} z+c_{j}\right)^{v_{j}}\right) \\
& \leq\{2+\min (2 d, \sigma)\} T(r, f)+S(r, f)
\end{aligned}
$$

In the same way,

$$
\begin{aligned}
& N_{2}\left(r, \frac{1}{F^{*}(z)}\right) \leq\{2+\min (2 d, \sigma)\} T(r, f)+S(r, f) \\
& N_{2}\left(r, G^{*}(z)\right) \leq\{2+\min (2 d, \sigma)\} T(r, g)+S(r, g) \\
& N_{2}\left(r, \frac{1}{G^{*}(z)}\right) \leq\{2+\min (2 d, \sigma)\} T(r, g)+S(r, g)
\end{aligned}
$$

Combining Lemma 2.4, Lemma 2.7, Equations ((21) and (22)), we obtain that

$$
\begin{aligned}
& (n-\sigma)(T(r, f)+T(r, g)) \leq T\left(r, F^{*}(z)\right)+T\left(r, G^{*}(z)\right) \\
& \leq 2 N_{2}\left(r, F^{*}(z)\right)+2 N_{2}\left(r, \frac{1}{F^{*}(z)}\right)+2 N_{2}\left(r, G^{*}(z)\right) \\
& +2 N_{2}\left(r, \frac{1}{G^{*}(z)}\right)+S\left(r, F^{*}(z)\right)+S\left(r, G^{*}(z)\right) \\
& \leq 4[2+\min (2 d, \sigma)](T(r, f)+T(r, g))+S(r, f)+S(r, g)
\end{aligned}
$$

Then

$$
(n-\sigma-8-4 \min (2 d, \sigma))(T(r, f)+T(r, g)) \leq S(r, f)
$$

Which is impossible, since $n \geq 4 \min (2 d, \sigma)+\sigma+9$.

Case 2. Suppose that $F^{*}(z) \equiv G^{*}(z)$ holds, we obtain

$$
f^{n}(z) \prod_{j=1}^{d} f\left(q_{j} z+c_{j}\right)^{v_{j}}=g^{n}(z) \prod_{j=1}^{d} g\left(q_{j} z+c_{j}\right)^{v_{j}} .
$$

We assume that $h(z):=\frac{f(z)}{g(z)}$. If $h(z) \equiv \mathbf{C} \quad$ (constant), then $f=t g$, and by substituting $f=\operatorname{tg}$ into (25), we obtain that

$$
g^{n} \prod_{j=1}^{d} g\left(q_{j} z+c_{j}\right)^{v_{j}}\left[t^{n+\sigma}-1\right]=0 .
$$

Since $g$ is a transcendental meromorphic function, than 
$g^{n} \prod_{j=1}^{d} g\left(q_{j} z+c_{j}\right)^{v_{j}} \not \equiv 0$. It follows that $t^{n+\sigma}=1$.

Suppose that $h(z) \not \equiv \mathbf{C}$ (constant), then using (25), we deduce that $h^{n}(z)=\prod_{j=1}^{d} \frac{1}{h\left(q_{j} z+c_{j}\right)^{v_{j}}}$,

So

$$
n T(r, h(z))=T\left(r, \prod_{j=1}^{d} \frac{1}{h\left(q_{j} z+c_{j}\right)^{v_{j}}}\right) \leq \sigma T(r, h(z))+S(r, h(z))
$$

We get a contradiction, since $n \geq 4 \min (2 d, \sigma)+\sigma+9$.

Case 3. Suppose that $F^{*}(z) G^{*}(z) \equiv 1$ holds, then

$$
f^{n}(z) \prod_{j=1}^{d} f\left(q_{j} z+c_{j}\right)^{v_{j}} \cdot g^{n}(z) \prod_{j=1}^{d} g\left(q_{j} z+c_{j}\right)^{v_{j}}=\alpha^{2}(z) \text {. }
$$

We define $h_{1}(z)=f(z) \cdot g(z)$, we easily get $h_{1}^{n}(z)=\prod_{j=1}^{d} \frac{\alpha^{2}(z)}{h_{1}\left(q_{j} z+c_{j}\right)^{v_{j}}}$ is non-constant, hence

$$
n T\left(r, h_{1}(z)\right)=T\left(r, \prod_{j=1}^{d} \frac{\alpha^{2}(z)}{h_{1}\left(q_{j} z+c_{j}\right)^{v_{j}}}\right) \leq \sigma T\left(r, h_{1}(z)\right)+S\left(r, h_{1}(z)\right)
$$

We get a contradiction, since $n \geq 4 \min (2 d, \sigma)+\sigma+9$. This implies that $h_{1}(z)$ is a constant, which is impossible.

\subsection{Proof of Theorem 1.2}

Set $F^{*}(z)=\frac{F(z)}{\alpha(z)}, G^{*}(z)=\frac{G(z)}{\alpha(z)}$, So $F^{*}(z)$ and $G^{*}(z)$ share 1 IM.

Using the same arguments as in Theorem 1.1, we prove that (18)-(22) holds.

We can easily get

$$
\begin{aligned}
& \bar{N}\left(r, F^{*}(z)\right) \leq(1+d) T(r, f)+S(r, f) \\
& \bar{N}\left(r, \frac{1}{F^{*}(z)}\right) \leq(1+d) T(r, f)+S(r, f) \\
& \bar{N}\left(r, G^{*}(z)\right) \leq(1+d) T(r, g)+S(r, g) \\
& \bar{N}\left(r, \frac{1}{G^{*}(z)}\right) \leq(1+d) T(r, g)+S(r, g)
\end{aligned}
$$

Let

$$
L(z)=\frac{F^{* \prime \prime}(z)}{F^{* \prime}(z)}-2 \frac{F^{* \prime}(z)}{F^{*}(z)-1}-\frac{G^{* \prime \prime}(z)}{G^{* \prime}(z)}+2 \frac{G^{* \prime}(z)}{G^{*}(z)-1}
$$

If $L \not \equiv 0$, combining Lemma 2.3, (21), (22) with (29), we obtain

$$
\begin{aligned}
& (n-\sigma)(T(r, f)+T(r, g)) \leq T\left(r, F^{*}(z)\right)+T\left(r, G^{*}(z)\right) \\
& \leq[14+6 d+4 \min (2 d, \sigma)](T(r, f)+T(r, g))+S(r, f)+S(r, g)
\end{aligned}
$$


Then,

$$
(n-\sigma-14-6 d-4 \min (2 d, \sigma))(T(r, f)+T(r, g)) \leq S(r, f)+S(r, g)
$$

that is impossible, since $n \geq 4 \min (2 d, \sigma)+\sigma+6 d+15$. Hence, we get $L \equiv 0$.

By integrating $\mathrm{L}$ twice, we obtain that

$$
F^{*}=\frac{(b+1) G^{*}+(a-b-1)}{b G^{*}+(a-b)}
$$

which yields $T\left(r, F^{*}\right)=T\left(r, G^{*}\right)+O(1)$. From Lemma 2.8, we deduced that $T(r, f)=T(r, g)+S(r, f)$. Next, we will consider the following three subcases.

Case 1. $b \neq 0$ and $b \neq-1$. Suppose that $a-b-1 \neq 0$, by (33), we get

$$
\bar{N}\left(r, \frac{1}{F^{*}}\right)=\bar{N}\left(r, \frac{1}{G^{*}-\frac{a-b-1}{b+1}}\right)
$$

Combining the second fundamental theory with Lemma 2.5, Lemma 2.7, (29), and (34), we have

$$
\begin{aligned}
& (n-\sigma) T(r, g) \leq T\left(r, G^{*}(z)\right)+S(r, g) \\
& \leq \bar{N}\left(r, G^{*}(z)\right)+\bar{N}\left(r, \frac{1}{G^{*}(z)}\right)+\bar{N}\left(r, \frac{1}{G^{*}-\frac{a-b-1}{b+1}}\right)+S(r, g) \\
& \leq \bar{N}\left(r, G^{*}(z)\right)+\bar{N}\left(r, \frac{1}{G^{*}(z)}\right)+\bar{N}\left(r, \frac{1}{F^{*}}\right)+S(r, g) \\
& \leq(2+2 d) T(r, g)+(1+d) T(r, f)+S(r, g) \\
& \leq(3+3 d) T(r, g)+S(r, g)
\end{aligned}
$$

which is impossible, since $n \geq 4 \min (2 d, \sigma)+\sigma+6 d+15$. Therefore, $a-b-1=0$, so

$$
F^{*}=\frac{(b+1) G^{*}}{b G^{*}+1}
$$

Then, $\bar{N}\left(r, \frac{1}{F^{*}}\right)=\bar{N}\left(r, \frac{1}{G^{*}+1 / b}\right)$. Similarly, we have

$$
\begin{aligned}
(n-\sigma) T(r, g) & \leq \bar{N}\left(r, G^{*}(z)\right)+\bar{N}\left(r, \frac{1}{G^{*}(z)}\right)+\bar{N}\left(r, \frac{1}{G^{*}+1 / b}\right)+S(r, g) \\
& \leq \bar{N}\left(r, G^{*}(z)\right)+\bar{N}\left(r, \frac{1}{G^{*}(z)}\right)+\bar{N}\left(r, \frac{1}{F^{*}}\right)+S(r, g) \\
& \leq(2+2 d) T(r, g)+(1+d) T(r, f)+S(r, g) \\
& \leq(3+3 d) T(r, g)+S(r, g)
\end{aligned}
$$

Which is impossible, since $n \geq 4 \min (2 d, \sigma)+\sigma+6 d+15$.

Case 2. If $b=0$ and $a=1$, then $F^{*} \equiv G^{*}$ obviously. From the proof of case 2 in theorem 1.1, we get $f(z)=\operatorname{tg}(z)$, where $t^{n+\sigma}=1$. Therefore, we 
consider $b=0$ and $a \neq 1$. Then from (33), we obtain

$$
F^{*}=\frac{G^{*}+a-1}{a} \text {. }
$$

Using the same discuss as Case 1, we get contradiction.

Case 3. If $b=-1$ and $a=-1$, then $F^{*} G^{*} \equiv 1$ obviously. Thus from the proof of case 3 in theorem 1.1, we get a contradiction. Therefore, we consider $b=-1$ and $a \neq-1$. From (33), we get

$$
F^{*}=\frac{a}{a+1-G^{*}} .
$$

Which is impossible, using the similar method as Case 1.

\subsection{Proof of Theorem 1.3}

We use the similar method as [14]. By the theorem condition that $F_{1}(z)-\alpha(z)$ and $G_{1}(z)-\alpha(z)$ share $0 \mathrm{CM}$, hence there exist an entire function $u(z)$, than

$$
\frac{F_{1}(z)-\alpha(z)}{G_{1}(z)-\alpha(z)}=\mathrm{e}^{u(z)}
$$

Since $\rho(f)=\rho(g)=0$, than $\mathrm{e}^{u(z)} \equiv \eta$ is a constant.

Rewriting (40)

$$
G_{1}(z)=F_{1}(z)+(\eta-1) \alpha(z)
$$

If $\eta \neq 1$, we can use Nevanlinnas two fundamental theorems, Lemma 2.5 and Lemma 2.8 to get a contradiction, since $n>\sigma+2 k+2 d$.

So we get $\eta=1$. Rewriting (40)

$$
\begin{aligned}
& P_{n}(f(z)) \prod_{j=1}^{d} f\left(q_{j} z+c_{j}\right)^{v_{j}} \\
& =P_{n}(g(z)) \prod_{j=1}^{d} g\left(q_{j} z+c_{j}\right)^{v_{j}} .
\end{aligned}
$$

Set $h(z):=\frac{f(z)}{g(z)}$, suppose that $h(z) \equiv \mathbf{C} \quad$ (constant), then $f=\operatorname{tg}$. Then we take $f=t g$ into (42) and get

$$
\prod_{j=1}^{d} g\left(q_{j} z+c_{j}\right)^{v_{j}}\left[\alpha_{n} g^{n}\left(t^{n+\sigma}-1\right)+\alpha_{n-1} g^{n-1}\left(t^{n+\sigma-1}-1\right)+\cdots+\alpha_{1} g\left(t^{\sigma+1}-1\right)\right] \equiv 0
$$

where $\alpha_{n}$ is a non-zero complex constant. And $\prod_{j=1}^{d} g\left(q_{j} z+c_{j}\right)^{v_{j}} \not \equiv 0$, since $g$ is transcendental meromorphic function. So $h^{m}=1$, where $m$ is greatest common divisor of $(n+\sigma, n+\sigma-1, \cdots, n+\sigma-i, \cdots, \sigma+1), \alpha_{n-i} \neq 0$ ( $i=0,1, \cdots, n-1)$.

Suppose that $h(z) \neq \mathbf{C}$ (constant), Equation (43) imply that $f(z)$ and $g(z)$ satisfy a algebraic equation $Q(f, g) \equiv 0$, where

$$
Q\left(w_{1}, w_{2}\right)=P_{n}\left(w_{1}\right) \prod_{j=1}^{d} w_{1}\left(q_{j} z+c_{j}\right)^{v_{j}}-P_{n}\left(w_{2}\right) \prod_{j=1}^{d} w_{2}\left(q_{j} z+c_{j}\right)^{v_{j}} .
$$




\section{Conclusion}

In this paper, we obtain some important results about the uniqueness of specific q-shift difference polynomials of meromorphic functions by Nevanlinna and value distribution theory and extend previous results. In addition, we also investigate the problem of value distribution on q-shift difference polynomials of entire functions.

\section{Acknowledgements}

Sincere thanks to the members of Xuexue Qian and Yasheng YE for their professional performance, and special thanks to managing editor for a rare attitude of high quality.

\section{References}

[1] Chen, Z.X. and Shon, K.H. (2010) Value Distribution of Meromorphic Solutions of Certain Difference Painlevé Equations. Journal of Mathematical Analysis \& Applications, 364, 556-556. https://doi.org/10.1016/j.jmaa.2009.10.021

[2] Chiang, Y. M. and Feng, S.J. (2008) On the Nevanlinna Characteristic of $f(z+\eta)$ and Difference Equations in the Complex Plane. Ramanujan Journal, 16, 105-129. https://doi.org/10.1007/s11139-007-9101-1

[3] Liu, K. and Yang, L.Z. (2009) Value Distribution of the Difference Operator. Archiv Der Mathematik, 92, 270-278. https://doi.org/10.1007/s00013-009-2895-x

[4] Yang, L.Z. and Zhang, J.L. (2008) Non-Existence of Meromorphic Solutions of a Fermat Type Functional Equation. Aequationes Mathematicae, 76, 140-150. https://doi.org/10.1007/s00010-007-2913-7

[5] Zhang, J. (2010) Value Distribution and Shared Sets of Differences of Meromorphic Functions. Journal of Mathematical Analysis \& Applications, 367, 401-408. https://doi.org/10.1016/j.jmaa.2010.01.038

[6] Zhang, J. and Korhonen, R. (2010) On the Nevanlinna Characteristic of $f(q z)$ and Its Applications. Journal of Mathematical Analysis \& Applications, 367, 537544. https://doi.org/10.1016/j.jmaa.2010.03.038

[7] Heittokangas, J., Korhonen, R., Laine, I., Rieppo, J. and Zhang, J. (2009) Value Sharing Results for Shifts of Meromorphic Functions, and Sufficient Conditions for Periodicity. Journal of Mathematical Analysis \& Applications, 355, 352-363. https://doi.org/10.1016/j.jmaa.2009.01.053

[8] Liu, K., Liu, X. and Cao, T.B. (2011) Value Distributions and Uniqueness of Difference Polynomials. Advances in Difference Equations, 2011, 1-12.

[9] Liu, K. and Qi, X.G. (2011) Meromorphic Solutions of q-Shift Difference Equations. Academic Press, 101, 1437-1444. https://doi.org/10.4064/ap101-3-2

[10] Chen, M.R. and Chen, Z.X. (2012) Properties of Difference Polynomials of Entire Functions with Finite Order. Chinese Annals of Mathematics, 33A, 359-374.

[11] Xu, H.Y., Liu, K. and Cao, T.B. (2015) Uniqueness and Value Distribution for q-Shifts of Meromorphic Functions. Mathematical Communications, 20, 97-112.

[12] Xu, J. and Yi, H. (2007) Uniqueness of Entire Functions and Differential Polynomials. Bulletin of the Korean Mathematical Society, 44, 623-629. https://doi.org/10.4134/BKMS.2007.44.4.623 
[13] Yang, L. (1982) Value Distribution Theory. Springer-Verlag, Berlin.

[14] Liu, Y., Cao, Y., Qi, X. and Yi, H. (2013) Value Sharing Results for q-Shifts Difference Polynomials. Discrete Dynamics in Nature and Society, 2013, Article ID: 152069.

[15] Yang, C.C. and Yi, H.X. (2003) Uniqueness of Meromorphic Functions. Kluwer, Dordrecht. https://doi.org/10.1007/978-94-017-3626-8

[16] Yang, C.C. and Hua, X. (1997) Uniqueness and Value-Sharing of Meromorphic

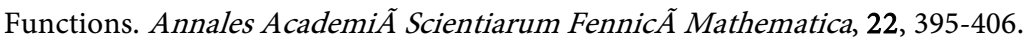

Submit or recommend next manuscript to SCIRP and we will provide best service for you:

Accepting pre-submission inquiries through Email, Facebook, LinkedIn, Twitter, etc. A wide selection of journals (inclusive of 9 subjects, more than 200 journals)

Providing 24-hour high-quality service

User-friendly online submission system

Fair and swift peer-review system

Efficient typesetting and proofreading procedure

Display of the result of downloads and visits, as well as the number of cited articles

Maximum dissemination of your research work

Submit your manuscript at: http://papersubmission.scirp.org/

Or contact am@scirp.org 